\title{
Overview of Initial Alignment Method for Strap down Inertial Navigation System
}

\author{
Hailiang Wang ${ }^{a}$, Guozhang Li and Zhiyong Shi \\ Ordnance Engineering College, Shijiazhuang 050003, China \\ awanghailiang17@126.com
}

Keywords: Strap down Inertial Navigation System, Coarse-Alignment, Fine-Alignment, TransferAlignment

\begin{abstract}
Initial alignment is one of the key technologies of Strap down Inertial Navigation System (SINS). The accuracy of initial alignment directly affect the accuracy of the work of SINS and the time of initial alignment are important tactical targets reflecting the rapid reaction capability of weapons systems. In this paper, the development and method of SINS initial alignment technology are reviewed from three aspects which include coarse alignment, fine alignment and transfer alignment.
\end{abstract}

\section{Introduction}

The navigation device needs to know its initial position, velocity and attitude information before entering the working condition. It is easy to know the initial position and velocity information, but the initial attitude information needs to be aligned. The so-called alignment refers to the process of determining the coordinates of the inertial navigation system with respect to the reference coordinate system.

The basic idea of initial alignment is that by processing the measured values of accelerometers and gyroscopes, the corrected angular velocity is generated for the updating calculation of the strap down matrix, and the misalignment angle is reduced to zero as much as possible. At the same time, the swing angular velocity of the carrier is calculated by measuring the output of the gyroscope, and the swing angular velocity of the carrier is separated. The schematic diagram is shown in the following figure.

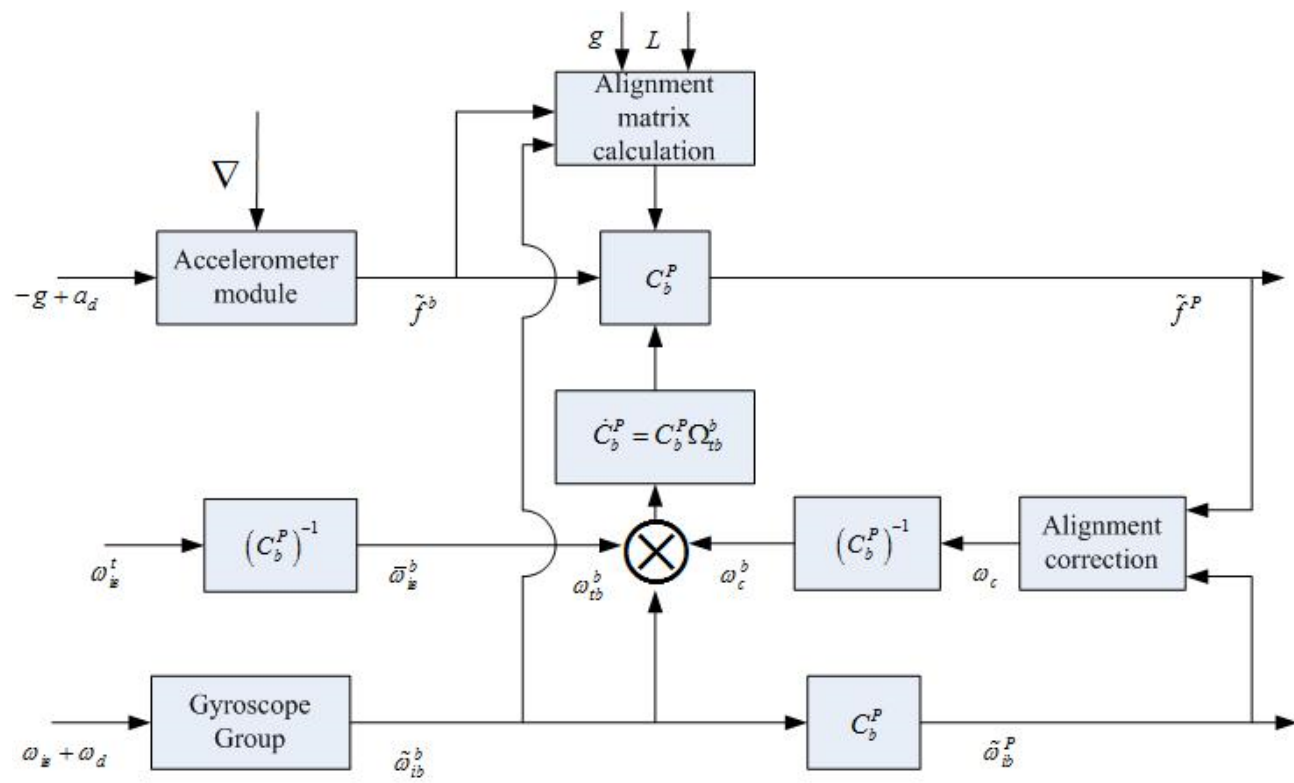

Fig. 1 Principle block diagram of initial alignment

Due to the existence of many complicated factors, the alignment of inertial navigation system are time-consuming and complicated. The precision of the initial alignment determines the precision of the navigation, so the research on the alignment technology of inertial navigation system has been paid much attention by many countries. At present, the initial alignment technology as a key 
technology of inertial navigation system has become the research hotspot and development direction in the field of navigation.

\section{Coarse-Alignment}

There are two main types of coarse alignment including coarse alignment of analytical method [1] and coarse alignment of inertial system. It is required to be able to meet the precise alignment of the initial misalignment angle as soon as possible to complete the alignment no matter which kind of coarse alignment method.

The basic principle of the coarse alignment is to determine the initial attitude matrix by using the gravity vector and the angular velocity vector of the earth. Under the condition of static state, the angular velocity measured by the gyro is the value of the rotation speed of the earth and the acceleration value is the value of gravity acceleration. When the carrier has a large swing motion, the earth's rotation speed information in the gyro measurements because of the signal-to-noise ratio is too low will not be available. When the carrier is in a large oscillatory motion, the measurement of acceleration will no longer be the gravity acceleration value. Therefore, the analytical method of coarse alignment is only applicable to the fully stationary base or the base of the interference motion.

In order to be able to align the movement base and the disturbance movement for the movement of the base, Gaiffe and Napolitano proposed a new method of coarse alignment [2-4], on the basis of the situations that the gravity vector is parallel to the earth, and the differential of the gravity vector and the east of the geographical system are parallel, this method completes the coarse alignment on inertial coordinate system. For this study, Qin Yongyuan [5] proposed a coarse alignment method based on gravity information for inertial system rocking base, the attitude matrix is decomposed into 4 matrices multiplication and then calculated respectively. Based on the characteristic of nonlinear phase of gravity vector at different time, and the gyro output tracking vector coordinate transformation of each movement, the transformation matrix of each inertial system is calculated, which can effectively solve the negative influence of the angular wobble on the coarse alignment. The algorithm will transform effective motor vector angle of oscillation, and the attitude update process to achieve separate diagonal movement, which can effectively isolate the interference angle of oscillation in the base, only when the angle of oscillation inertial coarse alignment accuracy and precision on the static base the coarse alignment of a cause[6], and the inertial system of coarse alignment line shaking also have certain inhibition effect, but it cannot completely eliminate the interference line shaking[7,8]. In order to reduce the influence of line shaking interference on the coarse alignment, many scholars have studied the method of eliminating the interference from the line by using the low-pass filter to the accelerometer measurement, and has achieved good results [9]. Naser El-Shimy et al. [10] proposed a method to remove high frequency noise by using multilevel wavelet decomposition. And Zhao Changshan [11] designed a polynomial least squares algorithm to eliminate line interference. On the basis of Qin Yangquan's research, an improved algorithm of inertial alignment was proposed by Yan Gongmin [12]. The algorithm takes into account the influence of the line motion on the initial alignment, and introduces the external velocity measurement information into the alignment, so that the carrier can complete the rough alignment between the moving.

Some scholars put forward the idea of using auxiliary equipment to introduce the external measurement information into the system to complete the coarse alignment. Liu Baiqi [13] used the uniform linear motion acceleration information for pitch angle and roll angle to improve the course information to complete the coarse alignment by Global Positioning System (GPS), but the method of coarse alignment has a larger error, and the carrier is in accordance with certain maneuver trajectory. She Haoping [14] used the GPS information to directly calculate the elevation angle and the heading angle, and then according to the kinematics equation of the vector around the center of mass, the least square method is used to estimate the roll angle. Qu Xinfen [15] introduced the GPS velocity information into the system, using the GPS velocity information to calculate the elevation angle and the heading angle, using the accelerometer and GPS velocity information to solve the roll angle, and the initial alignment, finally she completed the initial alignment. Ma Longhua [16] 
decomposed the calculation of attitude matrix as three independent transformation matrix to be solved, and made the measurement values of GPS and Inertial Measurement Unit (IMU) as the formation of integration to express in different coordinate systems in and out, so as to obtain the transformation matrix between different coordinate system to complete the coarse alignment. Bao Qilian [17] designed the initial alignment scheme of the electronic compass assisted accelerometer: first use the electronic compass measure the initial heading angle, and then use the accelerometer output calculate the initial roll angle and the initial pitch angle. However, the scheme requires the accelerometer to measure very accurately, and the calculation of the roll angle and the elevation angle will be greatly affected by the larger line interference. In the coarse alignment from the the horizontal angle of the Sun Wei [18], the attitude angle is calculated by the output of the accelerometer, and then the attitude angle is calculated by the output of the magnetometer. But the accuracy of the scheme is also limited by the accuracy of the accelerometer.

\section{Fine-Alignment}

The precise alignment is performed on the basis of coarse alignment, and the misalignment angle between the navigation coordinate system and the real navigation coordinate system is corrected to make it as close to zero as possible. Precision alignment requires the system to be completed as quickly as possible on the basis of accuracy.

In the early 1960s, Kalman filter theory was applied to the inertial navigation system, which provides a unified method for the initial alignment of the inertial navigation system, the velocity damping and the rest of the position. In 1965, L.D. Brock [19] used the Kalman filtering algorithm to deal with the initial alignment and calibration of the platform inertial navigation system. In 1967, J.F. Bellantoni [20] used Kalman filter with initial alignment and calibration of strap down inertial navigation system. When the misalignment angle is small, the error equation of SINS can be approximated as a linear equation, and the initial alignment can be achieved by two position alignment, multi position alignment and Kalman filter. At present, the initial alignment technique based on linear equation is relatively mature.

When the carrier is in situation that bad environment, or its attitude changes, the use of the coarse alignment of strap down attitude matrix error, error propagation angle error equation of the system cannot describe accurately the inertial navigation system based on a small loss, it is necessary to draw large misalignment angle error equations with nonlinear error response in initial alignment. At present, the nonlinear filtering method is used to study the initial alignment technique under the condition of large misalignment angle. Since 1980s, nonlinear filtering has been applied to the alignment of inertial navigation systems, such as Extended Kalman Filter (EKF), Unscented Kalman Filter (UKF), Particle Filter (PF), etc. EKF is a kind of approximate nonlinear filters, the basic idea is to linearize the nonlinear system, and then the Kalman filter, so EKF is a suboptimal filtering, and EKF is simple and easy to implement, which has been used in many disciplines and engineering fields. UKF is a nonlinear filtering method using strategy to approximate nonlinear distribution. So far, a number of domestic and foreign literature has been applied to inertial navigation system of the static base initial alignment and alignment [21-23], the use of speed matching algorithm, which has got a good estimation effect. In the late 1990s, PF also began to use in the initial alignment of inertial navigation system. The solution of PF is closest to the solution of Bayesian filtering, which has high estimation accuracy and is suitable for arbitrary distribution and any form of system. At the same time, the research of nonlinear filtering algorithm is developing towards the direction of Engineering application, more emphasis on the stability and real-time filtering algorithm. In order to solve the problem of particle degeneracy and sample depletion in PF and the large amount of calculation, people began to study the new algorithm of local linear particle filter, such as extended Kalman particle filter and unscented particle filter. At the same time, the adaptive algorithm, genetic algorithm and particle filter are combined to improve the filtering performance and reduce the computational complexity. 


\section{Transfer-Alignment}

Transfer alignment technology is also a kind of initial alignment, which can also be divided into two stages: coarse alignment and fine alignment. In the coarse alignment stage, the main inertial navigation system transfers the navigation information such as velocity and attitude to the inertial navigation system. In the fine alignment phase, the inertial navigation system (INS) is used to estimate and compensate the navigation information, such as the velocity and attitude of the main inertial navigation system. But because of lever arm effect, deflection and time delay error source, alignment error will appear, fully considering these factors which should be considered in the alignment process, and then we set up the mathematical model and make the corresponding compensation, so as to improve the accuracy.

Generally speaking, according to the different measurement methods in the transfer alignment, the method can be divided into two kinds: the calculation parameter matching method and the measurement parameter matching method. Calculation of parameter matching method is to use the main inertial navigation parameters and navigation parameters of missile system calculated the inertial navigation system is calculated to match, and measurement parameter matching method is the direct use of the main inertial navigation system and inertial navigation system output measurement to the matching process. From the perspective of the alignment precision, calculating parameter matching needs to be calculated and then navigation parameters are matched, so the calculating parameter matching alignment accuracy ratios measurement parameter matching alignment accuracy. But from the view of alignment time point, since the measurement parameter matching directly used the measured information navigation system and inertial navigation system of the main system, the calculation time matching calculation parameters omitted, so the alignment time of measurement parameters matching method than calculating parameter matching alignment time is short. At present, the matching methods are commonly used, such as speed matching, angular velocity matching, attitude matching method, angular velocity plus attitude matching method, velocity plus angular rate matching method and the velocity plus attitude matching method, etc., the velocity plus attitude matching method is the most widely used matching method.

\section{Summary}

From the analysis of research status of inertial navigation system initial alignment, the main purpose of the research is to improve the initial alignment of inertial navigation system accuracy and shorten the alignment time, and also can effectively further improve the accuracy of weapons and flexibility, strengthen land vehicles, aircraft, ship survivability and assault ability. At present, the initial alignment technology of inertial navigation system has been studied for many years in China. The research in this field has developed rapidly in recent years, but there are still some gaps. With the increase of various error model algorithms, the precision of INS is improved significantly. The future research focus of initial alignment technology should be in the exploration of improving the alignment accuracy and shorten the alignment time method, derived more effective alignment algorithm of inertial navigation system error model and the initial, these methods want to improve the precision of initial alignment. At the same time, the algorithm needs constant innovation.

\section{References}

[1]. Gao Wei, Ben Yue-yang, Li Qian. Initial Alignment Technique of Strapdown Inertial Navigation System[M]. Beijing: National Defence Industry Press, 2014.

[2]. Gaiffe T. From R\&D brassboard to navigation grade FOG-Based INS:the experience of Photonetics/Ixsea. Presented at IEEE, Optical Fiber Sensors Conference Technical Digest, 2002: $1-4$. 
[3]. Gaiffe T, Cottreau Y, Faussot N, et al. Highly compact fiber optic gyrocompass for applications at depths up to 3000 meters. Presented at IEEE/underwater technology, proceeding of the 2000 international symposium, 2000:155-160.

[4]. Napolitano F, Gaiffe T, Cottreau Y, et al. PHINS: the First high performances inertial navigation system based on fiber optic gyroscopes. Saint Petersburg, Russia: Presented at 9th saint Petersburg international conference on intergreted navigation systems, 2002: 296-304.

[5]. Qin Yong-yuan, Yan Gong-min, Gu Dong-qing, et al. A Clever Way of SINS Coarse Alignment despite Rocking Ship[J]. Journal of Northwestern Polytechnical University, 2005, 23(5): 681684.

[6]. Guo Xiao-song, Zhang Dong-fang, Xue Hai-jian, et al. Error Analysis and Scheme Optimization of Generalized Analytic Coarse Alignment[J]. Piezoelectrics \& Acoustooptics, 2015, 37(5): 918922.

[7]. Ma Yu. Research on Coarse Alignment of a Ship’s Strapdown Inertial Navigation System[D]. Harbin: Harbin Engineering University, 2014.

[8]. Shan Bin, Qin Yong-yuan, Zhang Jin-liang, et al. Anti-vibration Method in Indirect Analytic Alignment[J]. Journal of Projectiles, Rockets, Missiles and Guidance, 2015, 35(5): 76-80.

[9]. Yan Gong-min, Bai Liang, Weng Jun, et al. SINS Anti-Rocking Disturbance Initial Alignment Based on Frequency Domain Isolation Operator[J]. Journal of Astronautics, 2011, 32(7): 14861490.

[10]. Naser El-Sheimy, Sameh Nassar. Wavelet de-noising for IMU alignment. IEEE Aerospace and Electronic Systems Magazine. 2004, 19(10): 32-39.

[11]. Zhao Chang-shan, Qin Yong-yuan, Wei Liang. A Gravity-Based Anti-Interference Coarse Alignment Algorithm[J]. Journal of Astronautics, 2010, 31(10): 2335-2339.

[12]. Yan Gong-min, Qin Yong-yuan, Wei Yu-xin, et al. New initial alignment algorithm for SINS on moving base[J]. Systems Engineering and Electronics, 2009, 31(3): 634-637.

[13]. Liu Bai-qi, Gong Xiao-lin, Fang Jian-cheng. In-flight self-alignment for airborne SINS/GPS based on GPS observation and model predict filter[J]. Journal of Chinese Inertial Technology, 2007, 15(5): 568-572.

[14]. She Hao-ping, Yang Shu-xing, Ni Hui. New Algorithms to Estimate Initial Roll Angle for Inflight Alignment of GPS/INS Guided Munitions[J]. Acta Armamentarii, 2011, 32(10): 12651270.

[15]. Qu Xin-fen, Li Shi-ling, Xu Lin. Roll Angle In-flight Initial Alignment Method of INS/GPS Integrated System[J]. Journal of Detection \& Control, 2015, 37(4): 58-61.

[16]. Ma Long-hua, Wang Kai-li. A Novel Coarse Alignment Method of Strapdown Inertial Navigation System on Moving Base[J]. Journal of Projectiles,Rockets,Missiles and Guidance, 2013, 33(3): 1-4.

[17]. Bao Qi-lian, Wu Zhou-jie. Initial alignment methods of low-cost SINS assisted by magnetic compass[J]. Journal of Chinese Inertial Technology, 2008, 16(5):513-517.

[18]. Sun Wei, Ding Wei, et al. Research on the Initial Alignment of MEMS INS Based on Wavelet De-Noising[J]. Chinese Journal of Sensors and Actuators, 2015, 28(6): 814-818.

[19]. Brock L D. Application of statistical estimation to navigation systems. MIT,1965.

[20]. Bellantoni J F, Dodge K W. A Square Root Formulation of the Kalman-Schmidt Filter. Journal of AIAA, 1967, 5(7). 
[21]. Zhang Wei-ming, Zhang Ji-wei, Fan Zi-jie, et al. Research of unscented Kalman filter in initial alignment of inertial navigation systems[J]. Systems Engineering and Electronics, 2007, 29(4).

[22]. Wang Ya-feng, Sun Fu-chun, Zhang You-an. A Method for Rapid Transfer Alignment Based On UKF. Presented at IEEE, IMACS Multiconference on Computational Engineering in Systems Applications, 2006:809-813.

[23]. Eun-Hwan Shin, El-Sheimy N. An unscented Kalman filter for in-motion alignment of lowcost IMU. Presented at IEEE, Position Location and Navigation Symposiun, 2004:824-836. 